\title{
Angiogenesis Biomarkers in Ischemic Stroke Patients
}

\author{
Aziza Alrafiah (D) \\ Ebtisam Alofi ${ }^{2}$ \\ Yasser Almohaya' \\ Abdullah Hamami ${ }^{1}$ \\ Talal Qadah' \\ Safa Almaghrabi ${ }^{2}$ \\ Nora Hakami' \\ Moafaq S Alrawaili ${ }^{3}$ \\ Haythum O Tayeb ${ }^{3}$ \\ 'Department of Medical Laboratory \\ Technology, Faculty of Applied Medical \\ Sciences, King Abdulaziz University, \\ Jeddah, Saudi Arabia; ${ }^{2}$ Department of \\ Physiology, Medical School, King \\ Abdulaziz University, Jeddah, Saudi \\ Arabia; ${ }^{3}$ Division of Neurology, \\ Department of Internal Medicine, Faculty \\ of Medicine, King AbdulAziz University, \\ Jeddah, Saudi Arabia
}

\begin{abstract}
Introduction: Stroke is a global health issue, and ischemic stroke is among the most common strokes affecting many people worldwide. Throughout ischemic stroke, various immune cells counter its effect by releasing cytokines, chemokines, and angiogenic molecules. These molecules can work as potential biomarkers in the diagnosis and monitoring of the progress of ischemic stroke. The current study investigated the use of angiogenic molecules as biomarkers in ischemic stroke patients.
\end{abstract}

Methods: The samples were obtained from twenty healthy subjects and nineteen patients with ischemic stroke. Multiplex assay was used to measure the serum levels of angiogenic biomarkers, including endoglin, VEGF-A, endothelin-1, G-CSF, and angiopoietin-2. All data were analyzed using an unpaired Student's $t$-test. Correlations between measured parameters were made using Pearson correlations.

Results: Angiopoietin-2, VEGF-A, endothelin-1, and endoglin levels in stroke patients were significantly higher compared to healthy controls. Nevertheless, G-CSF level showed a nonsignificant increase in patients compared to controls. The correlation coefficient of measured angiogenic biomarkers among patients showed significant correlations between endoglin, angiopoietin, VEGF-A, and endothelin-1.

Discussion: The angiogenic factors were significantly increased in patients with ischemic stroke, which may help in the early detection of ischemic stroke and consequently prompt treatment and better prognosis.

Keywords: stroke, angiopoietin biomarkers, angiopoietin, endoglin, endothelin-1, VEGF-A

\section{Introduction}

Ischemic stroke is among the most common types of reported cases where disabilities or death are frequently observed. Inflammation is considered the primary concern and pathological constituent of the disease. ${ }^{1}$ Neuro-inflammatory processes significantly affect the nervous system by regulating brain cells and their connections development, maintenance, and sustenance. Microglia protects the nervous system by acting as scavengers of microbial pathogens and debris and regulating adaptive and innate immune responses. Pathological conditions, including ischemic stroke, injury, ${ }^{2}$ and infection, ${ }^{3}$ can lead to microglia activation. ${ }^{4}$ Activated microglia mediate glial cell and neuronal damage and death by generating proinflammatory factors like chemokines and cytokines and reactive oxygen species and support mobilizing the adaptive immune response. ${ }^{5}$

In cerebral ischemia, various immune cells responsible for angiogenesis are recruited and activated, resulting in vascular formation. ${ }^{6,7}$ The neurogenesis and angiogenesis processes are connected and coordinated after stroke. For instance, there is a movement of neuroblasts from the subventricular zone (SVZ) part to the infarct boundary, where there is an occurrence of post-stroke angiogenesis, and the
Correspondence: Aziza Alrafiah Tel +966 0126401000 Ext. 23495

Email aalrafiah@kau.edu.sa 
neuroblasts migrate through the cerebral vessels. ${ }^{8}$ The angiogenic process requires a synergistic effect of angiogenic growth factors and inhibitors to maintain the migration of endothelial cells (ECs) and proliferation necessary for repairing tissues over a specified period. ${ }^{9-11}$

Vascular endothelial growth factor A (VEGF-A) is a primary cerebral angiogenesis mediator and increases following a stroke in humans. ${ }^{12}$ Neural cells secreted VEGF-A when they were subjected to stroke or hypoxic conditions. VEGF production is widely associated with the adaptive form of the immune response to stroke or hypoxia, where promotes angiogenesis in post-stroke events, which leads to recovery of functions. ${ }^{13}$ Moreover, angiopoietin-2 (Ang 2) is known as angioneurotic, and, ${ }^{14}$ in a stroke patient, exogenous Ang2 acts as a protective factor as its level rises during the early stages of stroke. ${ }^{15}$ It also reduces the BBB permeability hence implying that Ang2 may be applicable as a biomarker in stroke. ${ }^{16}$

Endoglin is expressed on the surface of the endothelial cells, ${ }^{17}$ and it is essential for angiogenesis and released upon vessel injury by stroke. ${ }^{18}$ Several studies have shown that endoglin is usually overexpressed after hypoxia or stroke. ${ }^{19,20}$ In addition, G-CSF enhances recovery from stroke by providing the neuroprotective or repair role in the early and late stages of stroke and also causes a reduction in the volume of stroke lesions. ${ }^{21}$ However, endothelins are potent vasoconstrictors and exert several physiological effects such as endothelial function regulation. $^{22,23}$ Various CNS cells produce ET-1, and it has been found that overexpression of ET-1 in astrocytes led to severe neurological deficits, and higher mortality in transient ischemia model mice and subarachnoid hemorrhage. $^{24,25}$

The present study aimed to investigate the serum level of the angiogenic biomarkers including angiopoietin-1, G-CSF, endoglin, endothelin-1, and VEGF-A in healthy individuals and patients with ischemic stroke and compare the results and determine whether these factors can be used as a potential biomarker for ischemic stroke.

\section{Materials and Methods}

\section{Ethical Approval}

The study was approved by the ethical committee of King Abdulaziz University Hospital (Reference No 532-17). Informed written consent was obtained from all participants. This study was conducted in accordance with the Declaration of Helsinki.

\section{Materials}

The Angiogenesis/Growth Factor Panel 1 AntibodyImmobilized Magnetic Beads kits (Anti-Human Angiopoietin-2 Bead (CatNo. HANGPT2-MAG), AntiHuman Endoglin Bead (CatNo. HENDGLN-MAG), Anti-Human Endothelin-1 Bead (CatNo. HET1-MAG), Anti-Human G-CSF Bead (CatNo. HAGGCSF-MAG), and Anti-Human VEGF-A Bead (CatNo. HVEGFMAG)) were purchased from Millipore Corp (USA). The standards (CatNo. HNDG2-8036-2), quality controls 1 and 2 (CatNo. HNDG2-6036-2), bead diluent (CatNo. LBD), detection antibodies (CatNo. HNDG2-1036-2), and streptavidin-phycoerythrin (CatNo. L-SAPE6) were purchased from Invitrogen.

\section{Sample Collection and Processing}

Venous whole blood was collected by venipuncture from twenty healthy people and nineteen ischemic stroke patients whose samples were collected in the morning within 24 to 48 hours after the diagnosis, all participants were not suffering from diabetes mellitus, hypertension, and hypercholesterolemia. Patients with active infections or inflammation and other related factors that may interfere with protein signaling profiles were excluded.

Blood was collected into $10 \mathrm{~mL}$ EDTA-coated or heparinized tubes. Processing of the obtained blood samples included centrifugation at $3400 \mathrm{rpm}$ in 15 minutes at $4{ }^{\circ} \mathrm{C}$ to avoid platelets' activation. The plasma supernatant was removed without causing disturbance to the clot, which was immediately aliquoted then frozen at $-20{ }^{\circ} \mathrm{C}$ in 30 minutes to avoid minimal protein degradation. The processed sample storage was at $-80{ }^{\circ} \mathrm{C}$ as the sample awaited the next step, which included the quantification of angiogenic biomarkers (Dunbar and Hoffmeyer 2013).

\section{Methods}

The angiogenic biomarkers concentrations in the serum were measured using multiplex xMAP technology on a Luminex 200 instrument with commercially available panels from Millipore Corporation (Billerica, MA, USA). The procedures were performed according to the manufacturer's instructions, and the control samples that were provided within the kits were assayed in each analysis, and samples were run in duplicate. In our study, the levels of VEGF-A, endoglin, endothelin-1, angiopoietin-2, and G-CSF were studied. The data were generated by the 3.1 Xponent software package (Luminex Corporation, USA). 


\section{Statistical Analysis}

The data obtained during the study were analyzed utilizing IBM SPSS Statistics for Windows, version 23 (IBM SPSS, IBM Corp., Armonk, N.Y., USA). Shapiro-Wilk test was utilized to evaluate normal value distribution. Collected value presented as mean \pm standard deviation (SD). Statistical comparisons were made by unpaired Student's $t$-test to determine significance between patients and control. Correlations coefficient was made between measured parameters using Person equation. $\mathrm{P}<0.05$ was considered statistically significant.

\section{Results}

\section{Comparison of Serum Levels of Angiopoietin and Endoglin}

The study participants' demographic characteristics, including age, and sex, were non-significantly different between ischemic stroke patients and the healthy participants (Table 1). Angiopoietin-2 serum levels in ischemic stroke patients were significantly higher than controls subjects (healthy vs patient; $3430.60 \pm 612.32 \mathrm{pg} / \mathrm{mL}$ vs 1684.56 $\pm 228.18 \mathrm{pg} / \mathrm{mL}, \mathrm{P}=0.020$ ) as shown in (Figure 1A). Furthermore, the difference in endoglin concentration in serum between ischemic stroke patients and healthy
Table I Demographic Data of Individuals Participating in the Study

\begin{tabular}{|l|r|r|r|}
\hline Parameters & Control (n= 9) & Patients (n= 10) & P value \\
\hline Age (years) & $58.22 \pm 3.43$ & $54.90 \pm 10.37$ & 0.493 \\
\hline Gender & & & 0.570 \\
Male & $6(66.7 \%)$ & $6(60 \%)$ & \\
Female & $3(33.3 \%)$ & $4(40 \%)$ & \\
\hline
\end{tabular}

participants was significant (healthy vs patient; 2023.35 $\pm 175.86 \mathrm{pg} / \mathrm{mL}$ vs $1449.00 \pm 101.63 \mathrm{pg} / \mathrm{mL}, \mathrm{P}=0.014$ ) (Figure 1B).

\section{Comparison of Serum Levels of Endothelin-I (A), VEGF-A (B) and G-CSF} The serum levels of endothelin-1 in ischemic stroke patients were significantly higher than the control subjects $(9.27 \pm 1.04 \mathrm{pg} / \mathrm{mL}$ versus $6.41 \pm 0.03 \mathrm{pg} / \mathrm{mL}, P=0.019)$ (Figure 2A). Similarly, the levels of VEGF-A in ischemic stroke patients were significantly elevated compared to the control subjects $(626.35 \pm 69.40 \mathrm{pg} / \mathrm{mL}$ versus 179.91 $\pm 22.28 \mathrm{pg} / \mathrm{mL}, P=0.001$ ) (Figure 2B). Whereas the serum levels of G-CSG in ischemic stroke patients were higher than the control subjects, the differences did not reach a significant level $(128.74 \pm 4.19 \mathrm{pg} / \mathrm{mL}$ versus $123.46 \pm 0.14 \mathrm{pg} / \mathrm{mL}, P=0.250$ ) (Figure $2 \mathrm{C}$ ).
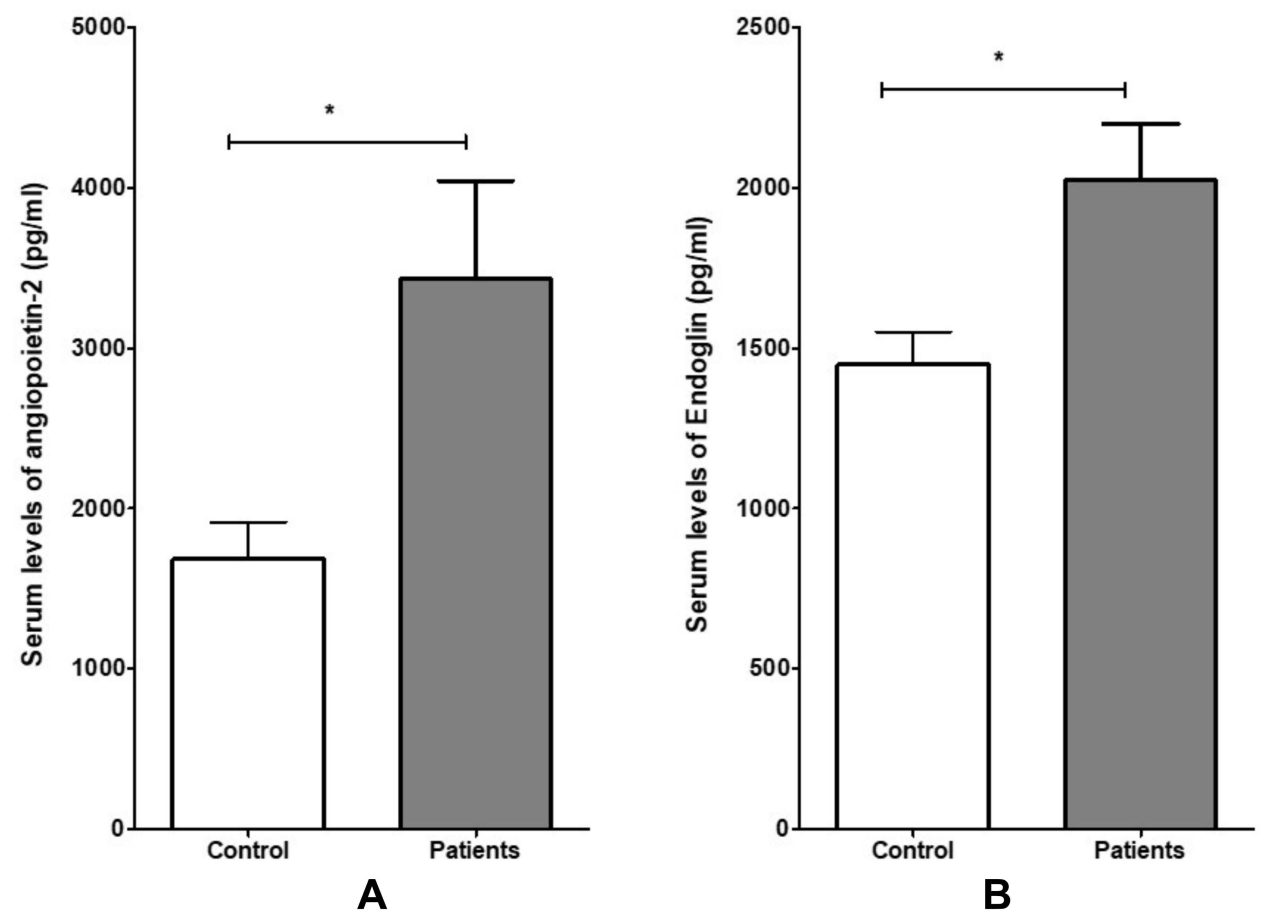

Figure I Comparison of serum levels of angiopoietin $(\mathbf{A})$ and endoglin $(\mathbf{B})$ results between patients and control. Data are expressed as mean \pm standard error. Difference between patients and control was made using unpaired Student's $t$-test for normally distributed data. $* P<0.050$. 


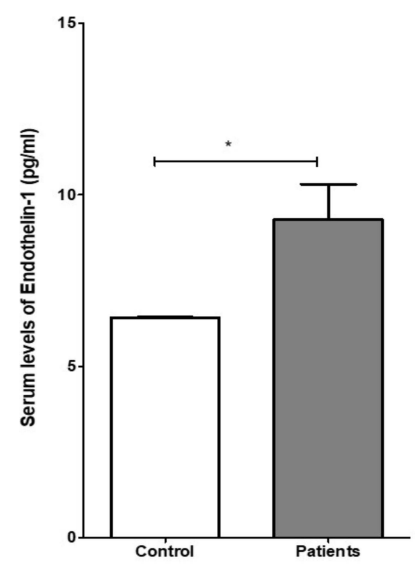

A

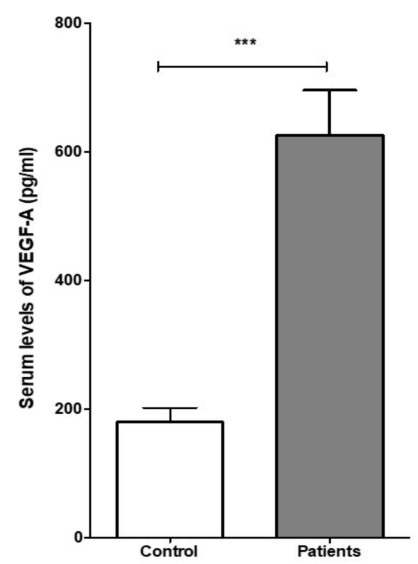

B

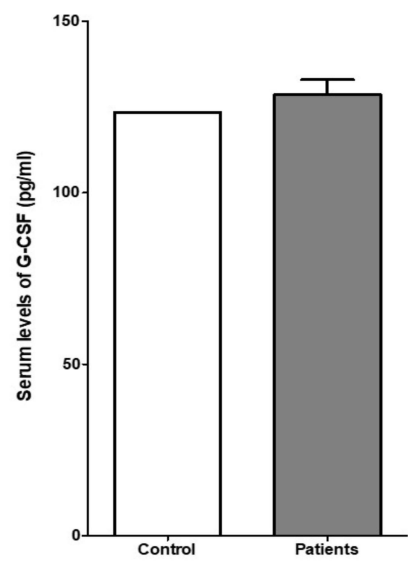

C

Figure 2 Comparison of serum levels of Endothelin-I (A), VEGF-A (B) and G-CSF (C) results between patients and control. Data are expressed as mean \pm standard error. Difference between patients and control was made using unpaired Student's $t$-test for normally distributed data. $* P<0.050$ and $* * * P<0.00$ I.

\section{Correlations Coefficient Between Serum} Levels of Angiopoietin and Endoglin Patients Correlations coefficients were computed among four angiogenic biomarkers on data of nine control persons; relatively, results indicated there were no significant correlations between measured angiogenic markers in the control group. In contrast, ten ischemic stroke patients' results data show a significant correlation and strong relationship between the four angiogenic biomarkers. In stroke patients, endoglin and endothelin-1 showed significant positive correlations $(\mathrm{r}=0.722 ; P=0.001)$ and $(\mathrm{r}=0.842 ; P=0.001)$, respectively with serum level parameter of angiopoietin-2 (Figure 3).

\section{Correlations Coefficient Between Serum Levels of Endoglin and Endothelin I and Between Serum Levels of Endothelin-I and VEGF in Patients}

Moreover, in patients, endothelin-1 and VEGF 1exhibited positive significant correlations $(\mathrm{r}=0.631 ; P=0.004)$ and $(\mathrm{r}=0.512 ; P=0.025)$ respectively with the serum levels of endoglin (Figure 4). Correlational analyses were used to examine the relationship between two variables, endothelin, and VEGF serum levels, showed a significant positive correlation ( $\mathrm{r}=0.509 ; P=0.026)$, a scatter plot summarizes the result (Figure 5), increase in endothelin was correlated with an increase in VEGF.

\section{Discussion}

Ischemic stroke is the most common type of stroke that occurs when the neck's or brain's blood vessel is blocked. Stroke significantly impacts public health because it is
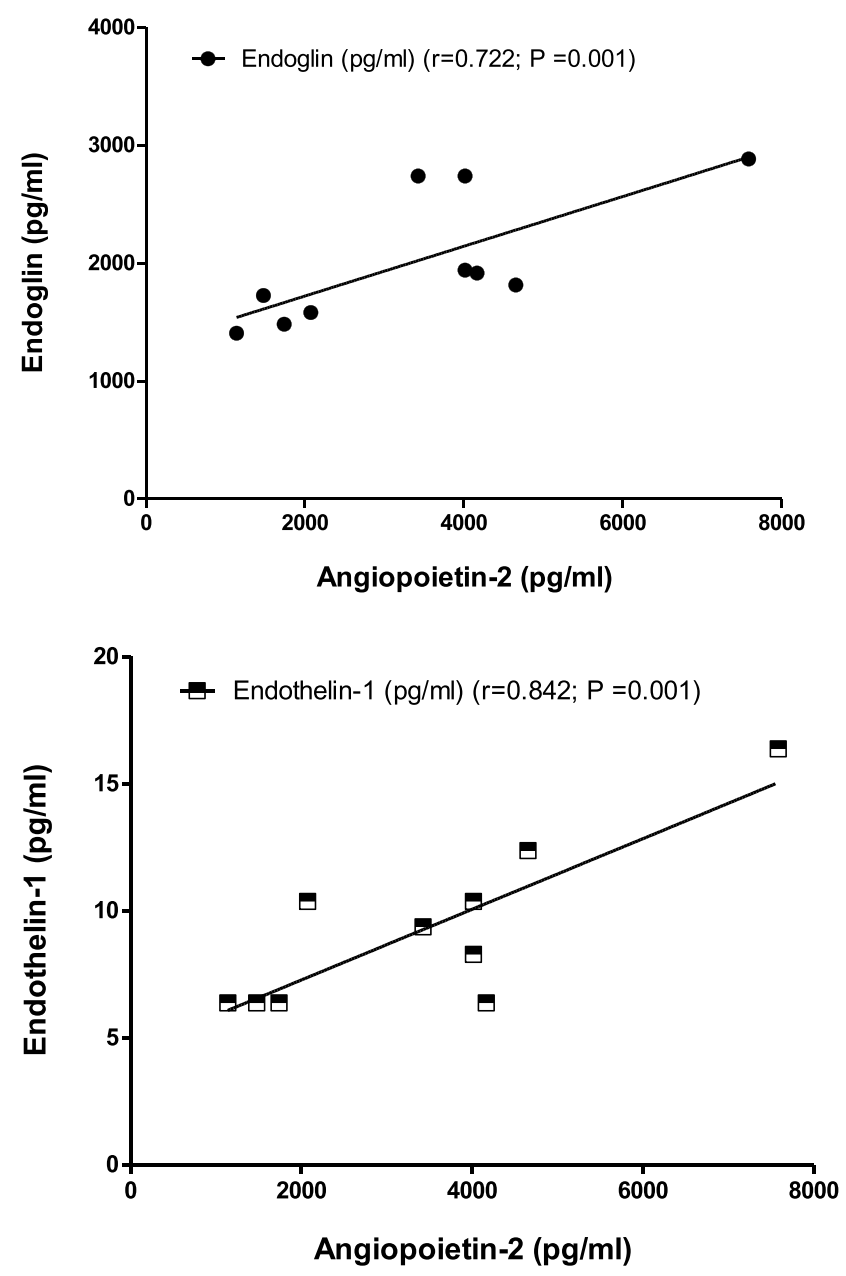

Figure 3 Correlations coefficient between serum levels of angiopoietin and endoglin patients $(r=0.722 ; P=0.00 \mathrm{I})$, and of angiopoietin and endothelin I in patients $(r=0.842 ; P=0.00 I)$ using Person test.

frequent, recurring, and is more often disabling than fatal. It is usually hard to distinguish between ischemic stroke and other medical conditions such as neurological 

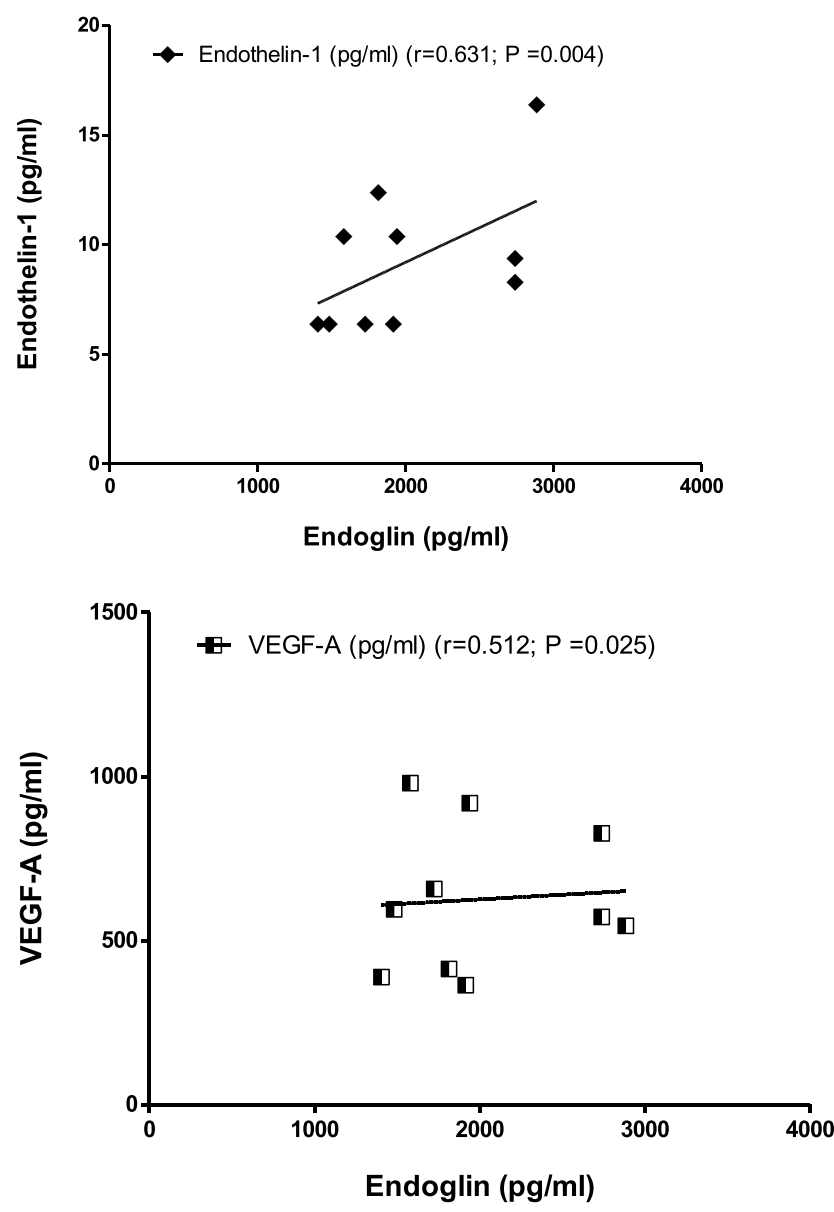

Figure 4 Correlations coefficient between serum levels of endoglin and endothelin $I$ in patients $(r=0.63 \mathrm{I} ; P=0.004)$, and endoglin and VEGF in patients $(r=0.5 \mathrm{I}$; $P=0.025)$ using Person test.

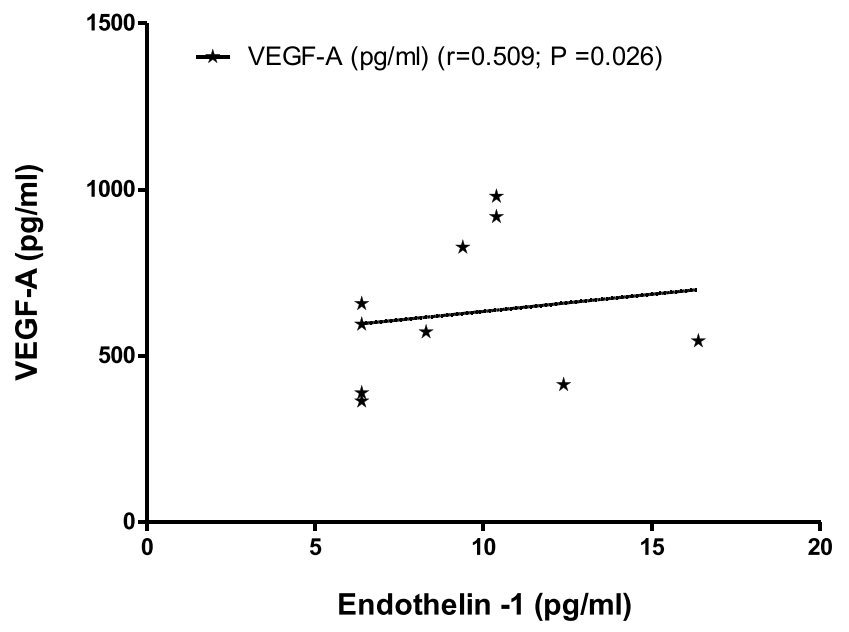

Figure 5 Correlations coefficient between serum levels of endothelin-I and VEGF in patients $(r=0.509 ; \mathrm{P}=0.026)$ using Person test.

disease despite both modifiable and non-modifiable risk factors identified for the disease in the presence of mild symptoms. ${ }^{26}$ It is, however, necessary to create a definite diagnostic model since instant diagnosis is a crucial factor in early intervention and recovery from the ailment.

This study aimed to identify critical biomarkers for ischemic stroke to enhance quick diagnosis; hence, early and appropriate intervention. One of the processes that occur after ischemic stroke is angiogenesis, caused by hypoxia and necessary for wound healing and embryogenesis following cerebral infarction. ${ }^{27}$ The angiogenic growth factors guide the migration and proliferation of endothelial cells necessary to repair the injured brain site. ${ }^{28,29}$ Therefore, some of the angiogenic mediators can be used as ischemic stroke biomarkers; several biomarkers can be selected to study ischemic stroke. This study focuses on the possibility of using makers from different families to offer predictive information. ${ }^{30}$

In the current research, we found that serum levels of angiopoietin in ischemic stroke patients were significantly higher than in the control group. Our results are similar to a study by Gurnik et al 2016, which showed that angiopoietin-2 increase BBB permeability and is associated with extended stroke size. ${ }^{31}$ The angiopoietin 1 and 2 molecules act as antagonists regulating angiogenesis, vascular permeability and stability, and lymphatic integrity. Stroke is associated with the disruption of BBB, and the ischemic stroke patients had an upregulated level of Angiopoietin-2. Thus, the results demonstrate that angiopoietin-2 is a vital biomarker of ischemic stroke, which mediates the permeability of the BBB. Angiopoietin-2 (Ang-2) is a growth factor that initiates downstream signaling via the Tie2 receptor tyrosine kinase. ${ }^{32,33}$ The endothelial cells predominantly express Tie 2 , which controls the maturation and remodeling of the vessels. ${ }^{34,35}$ While Ang-1 phosphorylates the Tie2 receptor to activate it, Ang-2 competitively binds to the receptor to prevent phosphorylation; thus, inactivating the Tie 2 and enabling angiogenesis. ${ }^{34,36}$ Comparing the control and the ischemic patients' group shows that the healthy adult brain does not express Ang-2 in its endothelium (REF). Therefore, the role of angiopoietin-2 in the pathophysiology of ischemic stroke is implicated in the study.

Our result showed that the endoglin serum levels are significantly higher in patients than in the control group; endoglin is a vital growth factor necessary for angiogenesis and vasculogenesis. It is also an essential component of the endothelial nitric oxide synthase activation complex. ${ }^{37}$ Endoglin can act as an important biomarker since animal studies have indicated its deficiency negatively impacts stroke recovery. High serum levels of 
endoglin could indicate positive disease progress, while low serum levels could be a potential indicator of regression. $^{37}$

We have found that endothelin-1 serum levels are significantly higher in patients with ischemic stroke. A similar study by Sapira et al 2010 showed that plasma endothelin-1 levels within the first 24 hours of stroke onset were significantly higher than in the control group. ${ }^{38}$ Ischemic stroke is therefore associated with a significant increase in the endothelin-1 levels in plasma. The findings may reflect on its increased production by the damaged endothelial cells remaining in the infarcted lesion. The biomarker can distinguish between ischemic stroke onset and other non-vascular ailments that present similar symptoms.

Moreover, our data showed that the ischemic patients have significantly higher VEGF-A serum levels than the healthy control group. Several previous studies demonstrate a correlation between VEGF-A upregulation and angiogenesis following middle cerebral artery occlusion. ${ }^{39-41}$ VEGF$\mathrm{A}$ is a primary cerebral mediator of angiogenesis which increases following a stroke in rodent models and humans. $^{42-45}$ Therefore, it can be a potential candidate as a biomarker for ischemic stroke. Also, since VEGF-A administration has been shown to enhance post-ischemic angiogenesis, ${ }^{46,47}$ the VEGF signaling pathways can be investigated as treatment targets for stroke.

Our result showed that the G-CSF serum levels are higher in the patients than in control, but the differences are insignificant. Previous research has shown that G-CSF may play an important role in protecting against ischemic brain injury in animal models and humans. The study by Yu et al 2012 is the first to compare the increase in endogenous G-CSF levels to the severity of the stroke and not simply the existence of an ischemic event. ${ }^{48}$ The results indicated that acute ischemic stroke led to a significant secretion of endogenous G-CSF levels and positively correlated with the severity of the condition on the first day after the incident. Endogenous G-CSF is, therefore, an excellent potential biomarker for assessing the severity of the condition. The current study results are inconclusive in determining the use of endogenous G-CSF as a biomarker in ischemic stroke since they demonstrate insignificant differences between a healthy control group and the patients. The results could indicate that the participants suffered less severe stroke ischemic stroke incidences. Future studies should consider using the biomarker on a large sample of individuals with varying severities of the condition to determine if less severe cases have insignificant G-CSF levels compared to healthy individuals.

The control group demonstrated no significant correlations between the angiogenic biomarkers, while the ischemic group patients had significant correlations between the biomarkers. The significant correlation shown in the current study can be used in future studies, which can use large participant numbers to approximate the correlation values of the biomarkers to determine the severity and presence of the disease.

The study's strength is in the ability of the Luminex technology to do multiple evaluations of several samples hence time and cost-effectiveness in analyzing multiple biomarkers at the same time. The exclusion of participants with preexisting conditions such as hypertension and diabetes, and other diseases that interfere with protein signaling also improved the viability of the results.

\section{Conclusions}

In conclusion, we have demonstrated that the angiopoietin-2, VEGF-A, endothelin-1, and endoglin serum levels increased significantly in patients with ischemic stroke compared to healthy volunteers. Furthermore, there a significant positive correlation between the studied angiogenic factors. These biomarkers could be beneficial to intensify clinicians' suspicion for the early diagnosis of ischemic stroke and, consequently, guide precise treatment and, hopefully, a better prognosis. Finally, the results of this study can form baseline information for future works where the relationship between the angiogenic biomarkers and their respective serum levels can be measured to create a potential predictive model that may enhance not only the diagnosis of ischemic stroke but also potential targets for drug delivery.

\section{Institutional Review Board Statement}

The study was conducted according to the guidelines of King Abdulaziz University and approved by the ethical committee of King Abdulaziz University Hospital (Reference No 532-17). Informed written consent was obtained from all participants.

\section{Informed Consent Statement}

Informed consent was obtained from all subjects involved in the study. 


\section{Acknowledgments}

The authors would like to thank "The International Conference and Addiction Psychiatry and Mental Health (Addiction 2019)" for accepting the abstract for presentation at the conference.

\section{Funding}

This research received no external funding.

\section{Disclosure}

The authors declare no conflict of interest.

\section{References}

1. Sacco RL, Kasner SE, Broderick JP, et al. An updated definition of stroke for the 21st century: a statement for healthcare professionals from the American Heart Association/American Stroke Association. Stroke. 2013;44(7):2064-2089. doi:10.1161/STR.0b013e318296aeca

2. Nilupul Perera M, Ma HK, Arakawa S, et al. Inflammation following stroke. J Clin Neurosci. 2006;13(1):1-8. doi:10.1016/j.jocn.2005. 07.005

3. De Chiara G, Marcocci ME, Sgarbanti R, et al. Infectious agents and neurodegeneration. Mol Neurobiol. 2012;46(3):614-638.

4. Ramesh G, MacLean AG, Philipp MT. Cytokines and chemokines at the crossroads of neuroinflammation, neurodegeneration, and neuropathic pain. Mediators Inflamm. 2013;2013:480739. doi:10.1155/ 2013/480739

5. Gendelman HE. Neural immunity: friend or foe? J Neurovirol. 2002;8(6):474-479. doi:10.1080/13550280290168631

6. Vidale S, Consoli A, Arnaboldi M, Consoli D. Postischemic inflammation in acute stroke. J Clin Neurol. 2017;13(1):1-9. doi:10.3988/ jen.2017.13.1.1

7. Marlier Q, Verteneuil S, Vandenbosch R, Malgrange B. Mechanisms and functional significance of stroke-induced neurogenesis. Front Neurosci. 2015;9:458. doi:10.3389/fnins.2015.00458

8. Ruan L, Wang B, ZhuGe Q, Jin K. Coupling of neurogenesis and angiogenesis after ischemic stroke. Brain Res. 2015;1623:166-173. doi:10.1016/j.brainres.2015.02.042

9. Chen J, Cui X, Zacharek A, Chopp M. Increasing Ang1/Tie2 expression by simvastatin treatment induces vascular stabilization and neuroblast migration after stroke. J Cell Mol Med. 2009;13(7): 1348-1357. doi:10.1111/j.1582-4934.2008.00380.x

10. Chen J, Zhang C, Jiang H, et al. Atorvastatin induction of VEGF and BDNF promotes brain plasticity after stroke in mice. $J$ Cerebral Blood Flow Metabol. 2005;25(2):281-290. doi:10.1038/sj.jcbfm. 9600034

11. Ding J, Cheng Y, Gao S, Chen J. Effects of nerve growth factor and Noggin-modified bone marrow stromal cells on stroke in rats. J Neurosci Res. 2011;89(2):222-230. doi:10.1002/jnr.22535

12. Seto SW, Chang D, Jenkins A, Bensoussan A, Kiat H. Angiogenesis in ischemic stroke and angiogenic effects of Chinese herbal medicine. J Clin Med. 2016;5(6):56. doi:10.3390/jcm5060056

13. Casscells W. Growth factor therapies for vascular injury and ischemia. Circulation. 1995;91(11):2699-2702. doi:10.1161/01.CIR. 91.11.2699

14. Zacchigna S, Lambrechts D, Carmeliet P. Neurovascular signalling defects in neurodegeneration. Nat Rev Neurosci. 2008;9(3):169-181. doi:10.1038/nrn2336

15. Talwar T, Srivastava MV. Role of vascular endothelial growth factor and other growth factors in post-stroke recovery. Ann Indian Acad Neurol. 2014;17(1):1-6. doi:10.4103/0972-2327.128519
16. Suwanwela N, Koroshetz WJ. Acute ischemic stroke: overview of recent therapeutic developments. Аnпи Rev Med. 2007;58(1):89-106. doi:10.1146/annurev.med.58.070605.115306

17. Conley BA, Smith JD, Guerrero-Esteo M, Bernabeu C, Vary CPH. Endoglin, a TGF-beta receptor-associated protein, is expressed by smooth muscle cells in human atherosclerotic plaques. Atherosclerosis. 2000;153(2):323-335. doi:10.1016/S0021-9150(00) 00422-6

18. Akagi K, Ikeda Y, Sumiyoshi Y, et al. Estimation of angiogenesis with anti-CD105 immunostaining in the process of colorectal cancer development. Surgery. 2002;131(1):S109-S113. doi:10.1067/msy.20 02.119361

19. Dziewulska D, Rafalowska J. Role of endoglin and transforming growth factor-beta in progressive white matter damage after an ischemic stroke. Neuropathology. 2006;26(4):298-306. doi:10.1111/ j.1440-1789.2006.00700.x

20. Sánchez-Elsner T, Botella LM, Velasco B, Langa C, Bernabéu C. Endoglin expression is regulated by transcriptional cooperation between the hypoxia and transforming growth factor- $\beta$ pathways. J Biol Chem. 2002;277(46):43799-43808. doi:10.1074/jbc.M207160200

21. England TJ, Sprigg N, Alasheev AM, et al. Granulocyte-Colony Stimulating Factor (G-CSF) for stroke: an individual patient data meta-analysis. Sci Rep. 2016;6:36567. doi:10.1038/srep36567

22. Salonia R, Empey PE, Poloyac SM. Endothelin-1 is increased in cerebrospinal fluid and associated with unfavorable outcomes in children after severe traumatic brain injury. $J$ Neurotrauma. 2010;27(10):1819-1825. doi:10.1089/neu.2010.1402

23. Cheng YW, Li WJ, Dou XJ, et al. Role of endothelin-1 and its receptors in cerebral vasospasm following subarachnoid hemorrhage. Mol Med Rep. 2018;18(6):5229-5236.

24. Yeung PKK, Shen J, Chung SSM, Chung SK. Targeted over-expression of endothelin-1 in astrocytes leads to more severe brain damage and vasospasm after subarachnoid hemorrhage. $B M C$ Neurosci. 2013;14(1):131. doi:10.1186/1471-2202-14-131

25. Lo AC, Chen AY, Hung VK, et al. Endothelin-1 overexpression leads to further water accumulation and brain edema after middle cerebral artery occlusion via aquaporin 4 expression in astrocytic end-feet. J Cerebral Blood Flow Metabol. 2005;25(8):998-1011. doi:10.1038/ sj.jcbfm.9600108

26. GBD 2016 Lifetime Risk of Stroke Collaborators. Global, regional, and country-specific lifetime risks of stroke, 1990 and 2016. $N$ Engl J Med. 2018;379(25):2429-2437. doi:10.1056/NEJMoa1804492

27. Arenillas JF, Sobrino T, Castillo J, Dávalos A. The role of angiogenesis in damage and recovery from ischemic stroke. Curr Treat Options Cardiovasc Med. 2007;9(3):205-212. doi:10.1007/s11936007-0014-5

28. Zhang ZG, Zhang L, Jiang Q, Chopp M. Bone marrow-derived endothelial progenitor cells participate in cerebral neovascularization after focal cerebral ischemia in the adult mouse. Circ Res. 2002;90 (3):284-288. doi:10.1161/hh0302.104460

29. Ghani U, Shuaib A, Salam A, et al. Endothelial progenitor cells during cerebrovascular disease. Stroke. 2005;36(1):151-153. doi:10.1161/01.STR.0000149944.15406.16

30. Lopez MF, Sarracino DA, Prakash A, et al. Discrimination of ischemic and hemorrhagic strokes using a multiplexed, mass spectrometry-based assay for serum apolipoproteins coupled to multi-marker ROC algorithm. Proteomics Clin Appl. 2012;6(34):190-200. doi:10.1002/prca.201100041

31. Gurnik S, Devraj K, Macas J, et al. Angiopoietin-2-induced blood-brain barrier compromise and increased stroke size are rescued by VE-PTP-dependent restoration of Tie2 signaling. Acta Neuropathol. 2016;131(5):753-773. doi:10.1007/s00401-016-1551-3

32. Augustin HG, Young Koh G, Thurston G, Alitalo K. Control of vascular morphogenesis and homeostasis through the angiopoietinTie system. Nat Rev Mol Cell Biol. 2009;10(3):165-177. doi:10. 1038/nrm2639 
33. Eklund L, Saharinen P. Angiopoietin signaling in the vasculature. Exp Cell Res. 2013;319(9):1271-1280. doi:10.1016/j.yexcr.2013.03.011

34. Reiss Y, Scholz A, Plate KH. The angiopoietin - tie system: common signaling pathways for angiogenesis, cancer, and inflammation. In: Schmidt MHH, Liebner S, editors. Endothelial Signaling in Development and Disease. New York: Springer New York; 2015:313-328.

35. Sato TN, Tozawa Y, Deutsch U, et al. Distinct roles of the receptor tyrosine kinases Tie-1 and Tie-2 in blood vessel formation. Nature. 1995;376(6535):70-74. doi:10.1038/376070a0

36. Maisonpierre PC, Suri C, Jones PF, et al. Angiopoietin-2, a natural antagonist for Tie2 that disrupts in vivo angiogenesis. Science. 1997;277(5322):55-60. doi:10.1126/science.277.5322.55

37. Zhu W, Ma L, Zhang R, Su H. The roles of endoglin gene in cerebrovascular diseases. Neuroimmunol Neuroinflam. 2017;4:19 9-210. doi:10.20517/2347-8659.2017.18

38. Sapira V, Cojocaru I, Lilios G, Grigorian M, Cojocaru M. Study of endothelin-1 in acute ischemic stroke. Roman $J$ Internal Med. 2010;48:329-332.

39. Abumiya T, Lucero J, Heo JH, et al. Activated microvessels express vascular endothelial growth factor and integrin $\alpha \mathrm{v} \beta 3$ during focal cerebral ischemia. J Cerebral Blood Flow Metabol. 1999;19 (9):1038-1050. doi:10.1097/00004647-199909000-00012

40. Zhang ZG, Zhang L, Tsang W, et al. Correlation of VEGF and angiopoietin expression with disruption of blood-brain barrier and angiogenesis after focal cerebral ischemia. $J$ Cerebral Blood Flow Metabol. 2002;22 (4):379-392. doi:10.1097/00004647-200204000-00002
41. Kovács Z, Ikezaki K, Samoto K, Inamura T, Fukui M. VEGF and flt. Expression time kinetics in rat brain infarct. Stroke. 1996;27 (10):1865-72; discussion 72-3. doi:10.1161/01.STR.27.10.1865

42. Mu D, Jiang X, Sheldon RA, et al. Regulation of hypoxia-inducible factor $1 \alpha$ and induction of vascular endothelial growth factor in a rat neonatal stroke model. Neurobiol Dis. 2003;14(3):524-534. doi:10. 1016/j.nbd.2003.08.020

43. Chen HH, Chien CH, Liu HM. Correlation between angiogenesis and basic fibroblast growth factor expression in experimental brain infarct. Stroke. 1994;25(8):1651-1657. doi:10.1161/01.STR.25.8.1 651

44. Liu HM. Neovasculature and blood-brain barrier in ischemic brain infarct. Acta Neuropathol. 1988;75(4):422-426. doi:10.1007/BF0 0687796

45. Krupinski J, Kaluza J, Kumar P, Kumar S, Wang JM. Role of angiogenesis in patients with cerebral ischemic stroke. Stroke. 1994;25(9):1794-1798. doi:10.1161/01.STR.25.9.1794

46. Zhang ZG, Zhang L, Jiang Q, et al. VEGF enhances angiogenesis and promotes blood-brain barrier leakage in the ischemic brain. $J$ Clin Invest. 2000;106(7):829-838. doi:10.1172/JCI9369

47. Sun Y, Jin K, Xie L, et al. VEGF-induced neuroprotection, neurogenesis, and angiogenesis after focal cerebral ischemia. J Clin Invest. 2003;111(12):1843-1851. doi:10.1172/JCI200317977

48. Yu S-C, Kuo C-L, Huang C-S, et al. Endogenous granulocyte colony-stimulating factor: a biomarker in acute ischemic stroke. Biomarkers. 2012;17(4):319-324. doi:10.3109/1354750X.2012.668 712
Journal of Inflammation Research

\section{Publish your work in this journal}

The Journal of Inflammation Research is an international, peerreviewed open-access journal that welcomes laboratory and clinical findings on the molecular basis, cell biology and pharmacology of inflammation including original research, reviews, symposium reports, hypothesis formation and commentaries on: acute/chronic inflammation; mediators of inflammation; cellular processes; molecular

\section{Dovepress}

mechanisms; pharmacology and novel anti-inflammatory drugs; clinical conditions involving inflammation. The manuscript management system is completely online and includes a very quick and fair peerreview system. Visit http://www.dovepress.com/testimonials.php to read real quotes from published authors. 